\title{
Novel therapeutics: response and resistance in ovarian cancer
}

\section{OPEN ACCESS}

\section{Correspondence to} Dr Dmitriy Zamarin, Memorial Sloan Kettering Cancer Center, New York, NY 10065, USA; zamarind@mskcc.org

Received 5 April 2019 Accepted 16 May 2019
Check for updates

(C) IGCS and ESGO 2019. Re-use permitted under CC BY-NC. No commercial re-use. Published by BMJ.

To cite: Zamarin D. Int J Gynecol Cancer 2019;29:s16s21.

Dmitriy Zamarin ${ }^{\odot}$

\begin{abstract}
Here we review the latest pre-clinical and clinical developments for treatment of ovarian cancer, presented at the American Association of Cancer Research/Rivkin Center Ovarian Cancer Research Symposium held at the University of Washington in September 2018. Abstracts and presentations pertaining to the 'Novel Therapeutics' session were reviewed and are summarized here. The session featured a keynote presentation from Dr Ursula Matulonis, who summarized the current state of the art of treatment of ovarian cancer, including recent clinical trials incorporating the use of novel agents, including poly-ADP-ribose polymerase (PARP) inhibitors, other DNA-damaging agents, vascular endothelial growth factor receptor inhibitors, mirvetuximab soravtansine, and immune checkpoint blockade. Dr Jung-Min Lee then summarized the rationale and the results of early studies for targeting cell cycle checkpoint kinases for anti-cancer therapy. Eight submissions were selected for oral presentations, and 36 abstracts were presented as posters. The topics covered a range of clinical and pre-clinical strategies and biomarkers, including immunotherapy, mechanisms of chemotherapy, and PARP inhibitor resistance, DNA-damaging agents, and other novel therapeutic strategies. Key studies have highlighted that resistance to chemotherapy and PARP inhibitors remain a major challenge in therapy of ovarian cancer. Cancer stem cells represent an important mechanism of chemoresistance and strategies to target these cells may be a pathway to prevention of ovarian cancer relapse. Advancement of novel therapeutics targeting DNA damage, cell metabolism, and endoplasmic reticulum present some of the novel strategies in the pipeline. Emerging compelling preclinical data with novel antibody-drug conjugates targeting various surface receptors in ovarian cancer alone and in combination with immune checkpoint blockade generate a strong enthusiasm for rapid translation of these strategies to clinic.
\end{abstract}

\section{INTRODUCTION}

Since the last symposium in 2016, we have seen a series of developments in ovarian cancer, with approval of several poly-ADP-ribose polymerase (PARP) inhibitors in different treatment settings as well new clinical trials using agents targeting DNA repair, oncogenic pathways, and the immune system. The Novel Therapeutics session focused on the studies that aim to delineate the mechanisms of resistance to existing agents, identify novel targets, and develop new treatment combinations.

\section{CURRENT STATE OF THERAPY OF OVARIAN CANCER AND NOVEL COMBINATIONS IN CLINICAL TRIALS}

Dr Ursula Matulonis gave a keynote lecture in this session, summarizing the state of the art of treatment of ovarian cancer and the results of recent clinical trials. Given the advances with PARP inhibitor development in ovarian cancer, she highlighted the multiple ongoing trials using PARP inhibitors in combination with other agents. These include PARP inhibitors in combination with agents targeting other mechanisms of homologous DNA repair, vascular endothelial growth factor and vascular endothelial growth factor receptor inhibitors, such as cediranib and bevacizumab, phosphatidylinositol-3-kinase inhibitors, and programmed cell death protein 1 (PD-1) inhibitors, some of which have demonstrated signals of activity in early clinical trials. ${ }^{1-3}$ She further summarized clinical data with folate receptor-targeting antibody drug conjugate mirvetuximab soravtansine, with a single agent response rate of $39 \%$ in non-heavily pre-treated patients. ${ }^{4}$ Combinations of mirvetuximab soravtansine with other agents, such as pembrolizumab, chemotherapy, and bevacizumab are ongoing. The responses appear to be enriched in the patients with medium/high folate receptor $\alpha$ expression, which will serve as a biomarker for future patient selection.

\section{TARGETING CELL CYCLE CHECKPOINTS AND DNA REPAIR MECHANISMS}

Cell cycle checkpoint kinases represent another target for therapy in ovarian cancer, particularly those cancers not carrying inactivating BRCA mutations. Dr Jung-Min Lee summarized the pre-clinical data and early clinical data behind prexasertib, an inhibitor of checkpoint kinase 1/2 in non-BRCA-mutant ovarian cancer. In pre-clinical models, the drug demonstrates synergistic activity when used in combination with olaparib in non-BRCA-mutant cell lines, probably through inhibition of RAD51-mediated homologous DNA repair. ${ }^{5} \mathrm{~A}$ phase $\mathrm{I} / \mathrm{ll}$ trial in 28 patients with advanced platinum-resistant non-BRCA-mutant ovarian cancer demonstrated a promising response 
rate of $33 \% .{ }^{6}$ Grade $3 / 4$ neutropenia was the most common adverse event, which interestingly was transient. Dr Lee further discussed other ongoing studies targeting DNA checkpoints alone or in combination, including combinations of checkpoint kinase 1 inhibitor and mammalian target of rapamycin inhibitors, and checkpoint kinase 1 inhibitors with PARP inhibitors, which are ongoing. Finally, she presented early clinical data with combinations of carboplatin with Wee1 inhibitors, demonstrating a promising response rate of $43 \%$ with 5.3 months' median progression-free survival.

\section{PARP INHIBITORS: RECENT FINDINGS, MECHANISMS OF RESISTANCE, AND NOVEL COMBINATIONS}

Dr Kathleen Moore and colleagues presented results of the QUADRA study, which evaluated single agent niraparib at $300 \mathrm{mg}$ daily in patients with high-grade serous ovarian cancer and three or more prior lines of therapy. A total of 463 patients were treated in the study and the results were assessed according to platinum sensitivity, BRCA status, and homologous recombination deficiency status. In 456 patients with measurable disease, the disease control rate was $49 \%$. In the primary efficacy population, defined as homologous recombination deficiency positive, the overall response rate was $29 \%$, with a disease control rate of $71 \%$. The most commonly observed grade 3 and higher adverse events were anemia (26.3\%) and thrombocytopenia (20.5\%). Niraparib thus demonstrated promising activity in this heavily pre-treated population, including platinum-resistant patients and some non-BRCA-mutant patients (NT-101).

Despite the promising response rate, this trial highlights the sad reality that PARP inhibition is not an option for the majority of patients, particularly those with platinum-resistant disease and BRCA wild-type status. Identification of biomarkers of primary and acquired resistance and strategies to minimize toxicity thus remain a high priority for further development of this class of agents.

Nanoparticles present an innovative and attractive strategy for drug delivery to tumors as a means of minimizing systemic toxicity. ${ }^{7}$ Dr Paige Baldwin and colleagues explored nanoparticle encapsulation of talazoparib, followed by intraperitoneal delivery, to minimize systemic toxicities of PARP inhibition. The resultant drug formulation was more efficient in suppressing tumor growth than the parental drug, with no obvious signs of toxicity (NT-87).

A number of studies uncovered several new mechanisms of PARP inhibitor resistance. ${ }^{8}$ Dr Neil Johnson presented an oral abstract describing a novel mechanism responsible for acquired PARP inhibitor resistance. BRCA1 mutations in the BRCA1 C-terminal domain normally lead to protein misfolding and degradation, promoting sensitivity to PARP inhibitors. Dr Johnson and colleagues have uncovered a mechanism, whereby retention of intron in a BRCA1 gene containing mutation in the BRCT domain results in intron translation and loss of BRCA1 C-terminal domain. This led to robust BRCA1 C-terminal expression and resistance to chemotherapy. Dr Lu Liu and colleagues demonstrated that short-term and chronic PARP inhibition can drive aldehyde dehydrogenase 1A1 expression, thus conferring stemness to the cancer cells, ${ }^{9}$ which eventually drives adaptive resistance to PARP inhibitors (NT-100). Finally, Dr Anniina Färkkilä used in vitro exposure to PARP inhibitors to characterize the mechanisms of PARP inhibitor resistance in BRCA1 mutant cells. The resultant resistance to PARP inhibitors was mediated by several mechanisms in different clones, highlighting the potential for heterogeneity in resistance on PARPi treatment (NT-92).

With identification of PARP inhibitor resistance mechanisms comes the rationale for combination therapies to overcome the resistance. Dr Marilyne Labrie and colleagues implemented a "window of opportunity" trial to study adaptive resistance to PARP inhibitors. Using reverse-phase protein array analysis, measuring the expression of over 300 proteins in pre- and post-treatment samples, the authors were able to develop an algorithm that could suggest potential combination partners for PARP inhibition in specific patients (NT-098).

Targeting DNA damage through several targets is a rational strategy to overcome PARP inhibitor resistance. ${ }^{10}$ Dr Erin George presented an oral abstract on the potential utility of ataxia telangiectasia and Rad3-related protein (ATR) inhibitors in combination with PARP inhibitors. They identified constitutive activation of the ATR/checkpoint kinase 1 pathway in PARP inhibitor and carboplatin-resistant cell lines. Treatment of such cell lines with an ATR inhibitor in combination with an ataxia-telangiectasia mutated inhibitor was synergistic both in vitro and in vivo. Interestingly, this approach was also effective in CCNE-amplified tumors, which are known to be highly resistant to therapy. Dr Anne Steino and colleagues have explored VAL-083 as a strategy to overcome platinum and PARP resistance. VAL-083 is a first-in-class DNA-damaging agent, which induces inter-strand DNA crosslinks leading to double-stranded DNA breaks. The authors examined VAL-083 in combination with PARP inhibitors both in homologous recombination-proficient and homologous recombination-deficient cell lines and demonstrated synergistic activity in both settings (NT-109). Dr Ludmila Szabova and colleagues evaluated another example of a DNA-damaging agent-non-camptothecin topoisomerase I inhibitors - in ovarian cancer. BRCA1/2- and PALB2-deficient cell lines were highly sensitive to the new compounds. Moreover, combination between the new compounds and olaparib was synergistic (NT-110). Dr Amber Yasmeen and colleagues explored polyADP-ribose glycohydrolase (PARG) as a potential strategy to sensitize ovarian cancer cells to DNA-damaging agents. PARG is responsible for poly-ADP-ribose catabolism which is synthesized by PARP. ${ }^{11}$ Faulty poly-ADP-ribose formation or disintegration inhibits single-strand break repair. The authors demonstrate that PARG is expressed in $30 \%$ of ovarian cancers in The Cancer Genome Atlas. Inhibition of PARG in ovarian cancer cell lines resulted in reduced cellular proliferation and migration and sensitized the cells to PARP inhibitors and cisplatin (NT-117). Dr Rashid Gabbasov and colleagues focused on targeting heat shock protein 90 , which plays a role in mediating maturation and stability of several key proteins required for DNA damage response. ${ }^{12}$ They demonstrate that targeted inhibition of heat shock protein 90 with ganetespib sensitizes BRCA1-null cell lines to the effects of talazoparib (NT-094).

A number of studies looked at other signaling pathways that might be targeted in combination with PARP inhibition. Dr Alicia Beeghly-Fadiel and colleagues have demonstrated that nuclear orphan receptor NR4A1/TR3 plays an important pro-growth and pro-survival role in ovarian cancer. ${ }^{1314}$ Inhibition of NR4A1 using a chemical antagonist or small interfering RNA knockdown resulted in tumor growth inhibition, and was synergistic with PARP inhibitor therapy (NT-88). Dr Takeshi Fukumoto and colleagues have shown that up-regulation 


\section{Review Article}

of Wnt/ $\beta$-catenin pathway in BRCA-mutant cancer cells results in PARP resistance. ${ }^{15}$ Interestingly, activation of the Wnt pathway was secondary to N6-methyladenosine modification of FZD10 mRNA. PARP inhibition and Wnt/ $\beta$-catenin inhibitor showed synergistic suppression of growth of PARP inhibitor-resistant cancer both in vitro and in vivo in a xenograft ovarian cancer mouse model (NT-93).

\section{OVERCOMING CHEMOTHERAPY RESISTANCE}

A number of studies have focused on the mechanisms of resistance to chemotherapy and potential strategies to overcome it. Dr Wa Xian and colleagues used ovarian cancer resection specimens to generate libraries of ovarian cancer stem cells. The authors showed that while the majority of these cells are killed by chemotherapy, a number of clones are resistant to treatment. The resistant clones were characterized by a gene expression profile that was distinct from that of the sensitive clones. A broad screen of small molecules against the resistant clones proved that these cells are also resistant to other chemotherapy drugs. However, a number of compounds were either directly cytotoxic or cytotoxic in combination with paclitaxel, presenting a potential strategy to eliminate the resistant clones with upfront therapy and prevent cancer recurrence (NT-115). Dr Allison Sharrow and colleagues explored ovarian cancer stem cells as a potential mechanism of chemotherapy resistance. ${ }^{9}$ Using aldehyde dehydrogenase 1 (ALDH1) as a marker of stemness in ovarian cancer cell lines, they demonstrated the increased resistance of populations with high levels of ALDH1 to chemotherapy. The authors further used gene expression analyses in these populations and identified several up-regulated pathways, including mTOR, FGF18, and CD47, which could be explored therapeutically (NT-107). In support of these findings, Dr Nuzhat Ahmed presented an oral abstract summarizing a proteomic analysis of chemo-naive and chemo-experienced ovarian cancer cells isolated from patients, demonstrating that chemotherapy results in enrichment of markers of cancer stem cells as well as alterations in pathways involved in DNA repair, immune recognition, cell cycle, and metabolism. This study provided important findings about potential mechanisms of chemotherapy resistance that may lead to a relapse in ovarian cancer and generated a rationale for potential novel combinations.

Dr Alex Cole and colleagues presented an oral abstract summarizing data on a new mechanism of chemotherapy resistance mediated by nuclear factor of activated T cells 3 (NFAT3), which is over-expressed in cancer stem cells. ${ }^{16}$ They showed that over-expression of NFAT3 promotes quiescent phenotype with $\mathrm{GO}$ arrest. While in vivo this results in tumor growth arrest, it also leads to chemoresistance. These data thus highlight the possibility that NFAT3 might be a potential mechanism of chemotherapy targeting and presents an attractive therapeutic target to be used in combination with chemotherapy.

Tumor hypoxia results in a number of biologic changes in the tumor cells and microenvironment. ${ }^{17}$ Dr Andrea Nieto-Veloza demonstrated that chemical induction of hypoxia in ovarian cancer cell line results in paclitaxel resistance, although no effect is seen on sensitivity to cisplatin (NT-102). These findings highlight that hypoxia-mediated chemotherapy resistance does not occur with all agents.

Several groups employed new compounds in combination with chemotherapy as potential chemotherapy sensitizers. Dr Amber Yasmeen and colleagues explored whether differential sequencing of
PARP inhibitors and chemotherapy might improve efficacy. Regardless of BRCA mutational status, exposure to PARP inhibition before chemotherapy resulted in efficient induction of apoptosis in vitro (NT-118). Dr Vermont Dia presented a potential strategy to overcome chemoresistance induced by transforming growth factor $\beta 1$ using BG-4. BG-4 is a bioactive peptide isolated from the seeds of Momordica charantia and exhibits anti-cancer properties. ${ }^{18}$ While addition of transforming growth factor $\beta 1$ to ovarian cancer cell lines in the presence of paclitaxel and cisplatin resulted in chemotherapy resistance, the resistance was ameliorated by addition of BG-4. This was accompanied by reversal of transforming growth factor $\beta 1$-induced epithelial to mesenchymal transition, suggesting a possible mechanism of BG-4 action (NT-91). Dr John Giannios presented a strategy of targeting chemoresistant ovarian cancer cells through the use of paired guide RNAs targeting microRNA-221/222, which are known to inhibit apoptosis. ${ }^{19}$ By encapsulating paired guide RNAs into pegylated nanosomes tagged with anti-EphA2 antibodies, the authors were able to deliver the RNAs into EphA2-expressing ovarian cancer cells. This strategy led to inhibition of microRNA-221/222 biogenesis and downstream pathways, resulting in reversal of chemoresistance (NT-95).

\section{TARGETING ENDOPLASMIC RETICULUM STRESS PATHWAY}

Cancer cells are characterized by endoplasmic reticulum stress and unfolded protein response, which contributes to cancer cell survival and resistance to stress caused by chemotherapy, hypoxia, and nutrient deprivation. ${ }^{20} \mathrm{Dr}$ Carlos Telleria and colleagues explored endoplasmic reticulum stress as a potential mechanism to target ovarian cancer cells. Treatment with anti-progestin mifepristone or HIV protease inhibitor induced endoplasmic reticulum stress in ovarian cancer cell lines. Combination of these drugs with the proteasome inhibitor bortezomib resulted in enhanced ovarian cancer cell death (NT-111). Similarly, Dr Yang Yang-Hartwich and colleagues explored the unfolded protein response pathway as a potential target for overcoming chemoresistance in ovarian cancer. Using a novel sulfonamide SF-Y3, the authors demonstrated inhibition of proliferation and induction of apoptosis in ovarian cancer cells. This effect was primarily seen in cancer cells with high levels of Bip1, a key chaperone protein in the endoplasmic reticulum, implicating its role in SF-Y3-induced unfolded protein response (NT-116).

\section{TARGETING METABOLIC PATHWAYS}

Advances in understanding of metabolism and biosynthetic pathway alterations in cancer cells are the rationale for selective targeting of these pathways as a means to improve the efficacy of standard therapies. Dr Adegbite Emmanuel and colleagues revealed preliminary results of in silico screening of inhibitors of lactate dehydrogenase, which plays a role in ovarian cancer metabolism ${ }^{21}$ (NT-86). Dr Manish Patankar and colleagues explored the use of oxidative phosphorylation inhibitors in ovarian cancer, given the mounting evidence that the mitochondrial pathway can also contribute to cancer cell metabolism. They showed that atovaquone is an efficient inhibitor of electron transport in ovarian cancer cells and leads to tumor growth inhibition in an ID8 tumor model. The authors highlighted several mechanisms of action of atovaquone, including production of free radicals and 
inhibition of ion transport resulting in loss of mitochondrial membrane potential (NT-103).

\section{ANTIBODY DRUG CONJUGATES}

Targeting of ovarian cancer surface molecules using antibody drug conjugates is a viable therapeutic strategy, best supported by the initial data from the phase I trial of mirvetuximab soravtansine, a folate receptor-targeting antibody drug conjugate, which demonstrated a $26 \%$ response rate in a heavily pre-treated patient population and a $39 \%$ response rate in patients with three or fewer lines of therapy. ${ }^{4}$ This generates a strong rationale for evaluation of analogous strategies targeting other surface molecules and for further optimization of folate receptor $\alpha$ targeting. Dr Venita De Almeida presented pre-clinical data with STR0-002, a novel antibody drug conjugate targeting folate receptor $\alpha$, which was optimized by selection of the antibody, drug linker, conjugation site, and drugantibody ratio that conferred the best pharmacological properties. The resultant drug exhibited high potency in vitro and in vivo, while exhibiting a high safety profile in toxicology studies (NT-90). Dr Chunsheng Li and colleagues presented an antibody drug conjugate strategy targeting CD248, which is expressed by over $90 \%$ of ovarian cancers. In pre-clinical models, the antibody was cytotoxic to cancer cells both in vitro and in vivo and induced infiltration of lymphocytes into tumors, highlighting a potential dual mechanism of action of this strategy with rationale for combination with immune checkpoint blockade (NT-99). Finally, Dr Wolf Wiedemeyer and colleagues explored SC-003, another antibody drug conjugate targeting dipeptidase 3 . By screening a patient-derived xenograft bank of ovarian cancers, the authors identified dipeptidase 3 as a common target in tumor-initiating cells. Treatment of dipeptidase 3 + patient-derived xenograft models with SC-003 resulted in efficient tumor regression. By using dipeptidase 3-expressing syngeneic mouse cells, the authors further showed that such a strategy can synergize with systemic programmed cell death protein 1 (PD-1) blockade (NT-113).

\section{IMMUNOTHERAPY}

Treatment with immune checkpoint blockade has been evaluated in epithelial ovarian cancer, but with disappointing response rates to date. ${ }^{22-25}$ These findings necessitate the development of rational combinations and identification of biomarkers that might predict response to PD-1/PD-L1 blockade. Dr Dmitriy Zamarin presented an oral abstract discussing the results of a phase II clinical trial evaluating the use of the PD-L1 inhibitor durvalumab in combination with folate receptor $\alpha$ vaccine TPIV200 in 27 patients with heavily pre-treated platinum resistant/refractory ovarian cancer. While the overall response rate was $4 \%$, similar to previously observed results with single agent PD-1/PD-L1 inhibitors, the study demonstrated a median overall survival of 21 months, which is better than the expected overall survival of $<1$ year in this patient population. ${ }^{26}$ There was evidence of potentially enhanced clinical benefit from subsequent chemotherapy in the patients after completion of immunotherapy, highlighting the rationale for the use of chemotherapy in combination with immune checkpoint blockade in this patient population. Dr Denise Cecile presented pre-clinical findings of insulin growth factor binding protein 2 vaccine in ID8-luciferase tumor models. The authors also employed a new method of antitumor assessment using multi-view multi-spectral imaging, allowing for more accurate tumor volume assessment. The study was able to demonstrate heterogeneity in metastatic tumor distribution and response, suggesting that this strategy may be a useful tool for evaluation of inter-tumoral heterogeneity in animal models.

\section{OTHER THERAPEUTIC STRATEGIES}

A number of abstracts discussed other novel targets and therapeutic modalities with potential application to ovarian cancer. Dr Karen Levy presented an oral abstract discussing pre-clinical data behind the use of a new radiation approach termed FLASH, which consists of short pulses of ultra-high dose radiation given in a single fraction. ${ }^{27}$ In a peritoneal model of ID8 ovarian cancer, this approach was effective in controlling tumors, while having no significant toxicity in comparison with conventional radiation. Dr Varatharasa Thiviyanathan and colleagues developed single-stranded nucleic acid aptamers with ability to recognize and bind ovarian cancer endothelial cells through annexin A2. Using this technology, the authors made an RNA/DNA nanoparticle capable of delivering doxorubicin to cancer cells in animal models. This strategy presents a potential mechanism for delivering drugs directly to the tumors while avoiding systemic toxicity (NT-112).

Several studies focused on the alterations common to high-grade serous ovarian cancer and other histologies. Missense mutations in p53 are the most common genetic alterations in ovarian cancer. ${ }^{28}$ Dr Satish Kumar Ramraj and colleagues used the p53 reactivator drug PRIMA-1 ${ }^{\text {MET }}$ in combination with SHetA2, a small molecule that inhibits mortalin (mitochondrial heat shock protein 70 ). ${ }^{29}$ The combination was synergistic in ovarian cancer cell lines with mutant and wild-type p53 and showed additive activity in p53-null cell lines (NT-104).

Clear cell carcinoma of the ovary represents a highly chemoresistant sub-type of ovarian carcinoma and frequently harbors mutations in ARID1A. ${ }^{30}$ Dr Shogo Shigeta and colleagues performed small interfering RNA screens against two ovarian clear cell carcinoma cell lines. A number of genes were identified as potential targets, including bromodomain BET family proteins BRD2 and BRD3. Knockdown of these proteins using RNA interference resulted in tumor growth inhibition (NT-108). Dr Shuai Wu and colleagues explored the mechanisms of acquired resistance to enhancer of zeste homolog 2 (EZH2) inhibition. In ARID1A-mutated cancer cells, the switch of the SWI/SNF catalytic sub-unit from SMARCA4 to SMARCA2 was the primary mechanism of resistance, leading to induction of anti-apoptotic genes such as BCL2. Use of BCL2 inhibitor ABT263 was able to overcome EZH2 inhibitor resistance and was synergistic with EZH2 inhibition in vivo (NT-114).

Epithelial to mesenchymal transition contributes to ovarian tumor metastasis and chemoresistance. ${ }^{31} \mathrm{Dr}$ Junming Yue and colleagues demonstrated that knockout of the metal regulatory transcription factor 1 results in inhibition of this transition, leading to reduced cell proliferation, migration, and invasion (NT-120). Dr Carmela Ricciardelli and colleagues explored all-trans retinoic acid as a means of inhibiting the annexin A2-S100A10 signaling pathway, which plays a role in ovarian cancer invasion and metastasis. ${ }^{32}$ Treatment of ovarian cancer cells with all-trans retinoic acid led to reduced cell 


\section{Review Article}

survival, proliferation, and invasion, although the mechanism was not always S100A10-dependent (NT-105). Dr Flavio Rizzolio and colleagues demonstrated that targeting of peptidyl prolyl cis-trans isomerase (Pin1), which controls different oncogenes and tumor suppressors, can inhibit tumor growth in mouse ovarian cancer model. ${ }^{33}$ Chemical or short hairpin RNA inhibition of Pin1 in ovarian cancer cell lines sensitized the cells to the effect of carboplatin (NT-106).

Several studies have characterized new compounds whose mechanisms of action are not yet not fully understood. Dr Alexandria Young and colleagues explored synthetic analogs of phyllanthusmin for cytotoxic activity in ovarian cancer cell lines. ${ }^{34}$ The most potent analog, PHY34, has nanomolar potency in high-grade serous ovarian carcinoma cell lines in vitro and displayed cytotoxic activity through late-stage autophagy inhibition and activation of apoptosis. The analog was also effective with intra-peritoneal administration in xenograft models (NT-119). Dr Arvinder Kapur and colleagues used fabclavine, a metabolite of Xenorhabdus budapestensis, and demonstrated that the compound inhibited ovarian cancer cell line proliferation and induced apoptotic cell killing at nanomolar concentrations (NT-096). Dr Powel Crosley and colleagues generated a TRAlL-expressing recombinant vaccinia virus and demonstrated its activity against granulosa cell tumor cell lines. Combination of the recombinant virus with procaspase-activating compound 1 resulted in further potentiation of Iytic activity (NT-89). Dr David Pepin presented the results of the effects of Mullerian inhibiting substance on ovarian cancer cells isolated from ascites. ${ }^{35}$ Using single cell RNA sequencing, their study uncovered a high degree of heterogeneity of expression of known and novel markers related to epithelial-mesenchymal states and stemness, both across patients and within patient samples. In addition, they uncovered some unexpected effects of Mullerian inhibiting substance on the immune cells in ascites. These findings will be important in understanding responses to this substance as it progresses through clinical development.

Finally, several groups presented early data on identification of novel compounds and pathways for targeting in ovarian cancer. Dr Hilary Kenny and colleagues used high throughput screening of small molecules against an ovarian cancer organotypic model that recapitulates features of ovarian cancer stroma. They identified three compounds - two targeting tyrosine kinases-which inhibited ovarian cancer adhesion, invasion, and growth (NT-97). Dr Einav Zmora and colleagues analyzed endothelial growth factorlike ligands from ascites of 43 patients with ovarian cancer and found that $86 \%$ of them expressed high levels of amphiregulin, which is a cytokine playing a role in tissue repair and inflammation. $^{36}$ The authors generated an antibody against amphiregulin and evaluated it in animal tumor models. Treatment with anti-amphiregulin resulted in significant prolongation of survival of mice bearing cancer xenografts (NT-121).

\section{CONCLUDING REMARKS}

In summary, the symposium presented a rich influx of information, highlighting novel mechanisms of primary and acquired resistance to chemotherapy and PARP inhibition. The revolving theme of combination therapy to overcome or prevent resistance resonates throughout all studies. Potential combination partners include other agents inhibiting DNA repair, agents targeting cellular checkpoints, and drugs effective against cancer stem cells. Immune checkpoint inhibitors, while effective in a small subset of patients, unfortunately demonstrate limited single-agent activity in ovarian cancer and rational combinations with other immunotherapies, PARP inhibitors, and standard chemotherapy are currently underway. Other novel therapeutic strategies focusing on endoplasmic reticulum stress response, epithelial to mesenchymal transition, and targeting of surface molecules using novel antibody drug conjugates demonstrate compelling evidence of anti-tumor activity in pre-clinical models and generate a strong rationale for evaluation in clinic.

Acknowledgements We thank Dr Deborah Armstrong for the critical review of the manuscript.

Contributors DZ was responsible for the review of the conference data and manuscript composition.

Funding DZ is a member of the Parker Institute for Cancer Immunotherapy at MSKCC. DZ is supported by the Ovarian Cancer Research Foundation Liz Tilberis Award, and the Department of Defense Ovarian Cancer Research Academy (0C150111). This work was supported in part by the MSK Cancer Center Support Grant P30 CA008748.

Competing interests DZ indicated financial activities outside of the submitted work, which include personal fees, grants, and intellectual property.

Patient consent for publication Not required.

Provenance and peer review Commissioned; internally peer reviewed.

Open access This is an open access article distributed in accordance with the Creative Commons Attribution Non Commercial (CC BY-NC 4.0) license, which permits others to distribute, remix, adapt, build upon this work non-commercially, and license their derivative works on different terms, provided the original work is properly cited, an indication of whether changes were made, and the use is non-commercial. See: http://creativecommons.org/licenses/by-nc/4.0/.

\section{REFERENCES}

1. Konstantinopoulos $\mathrm{P}$, Munster P, Forero-Torres A, et al. TOPACIO preliminary activity and safety in patients (PTS) with platinumresistant ovarian cancer in a phase 1/2 study of niraparib in combination with pembrolizumab. New Orleans, LA: SGO Annual Meeting, 2018.

2. Liu JF, Barry WT, Birrer M, et al. Combination cediranib and olaparib versus olaparib alone for women with recurrent platinum-sensitive ovarian cancer: a randomised phase 2 study. Lancet Oncol 2014;15:1207-14.

3. Konstantinopoulos PA, Barry W, Birrer M, et al. Phase I study of the alpha specific PI3-kinase inhibitor BYL719 and the poly (ADPribose) polymerase (PARP) inhibitor olaparib in recurrent ovarian and breast cancer: analysis of the dose escalation and ovarian cancer expansion cohort. Cancer Res 2017;77(13 Suppl). Abstract nr CT008.

4. Moore KN, Martin LP, O'Malley DM, et al. Safety and activity of mirvetuximab soravtansine (IMGN853), a folate receptor alphatargeting antibody-drug conjugate, in platinum-resistant ovarian, fallopian tube, or primary peritoneal cancer: a phase I expansion study. J Clin Oncol 2017;35:1112-8.

5. Brill E, Yokoyama T, Nair J, et al. Prexasertib, a cell cycle checkpoint kinases 1 and 2 inhibitor, increases in vitro toxicity of PARP inhibition by preventing Rad51 foci formation in BRCA wild type high-grade serous ovarian cancer. Oncotarget 2017;8:111026-40.

6. Lee J-M, Nair J, Zimmer A, et al. Prexasertib, a cell cycle checkpoint kinase 1 and 2 inhibitor, in BRCA wild-type recurrent high-grade serous ovarian cancer: a first-in-class proof-of-concept phase 2 study. Lancet Oncol 2018;19:207-15.

7. Calzoni E, Cesaretti A, Polchi A, et al. Biocompatible polymer nanoparticles for drug delivery applications in cancer and neurodegenerative disorder therapies. J Funct Biomater 2019;10.10.3390/jfb10010004

8. Lim J, Tan DSP. Understanding resistance mechanisms and expanding the therapeutic utility of PARP inhibitors. Cancers 2017;9. 
9. Shah MM, Landen CN. Ovarian cancer stem cells: are they real and why are they important? Gynecol Oncol 2014;132:483-9.

10. Pilie PG, Tang C, Mills GB, et al. State-of-the-art strategies for targeting the DNA damage response in cancer. Nat Rev Clin Oncol 2018;16:81-104.

11. Bu X, Kato J, Moss J. Emerging roles of ADP-ribosyl-acceptor hydrolases (ARHs) in tumorigenesis and cell death pathways. Biochem Pharmacol 2018.10.1016/j.bcp.2018.09.028

12. Pennisi R, Ascenzi P, di Masi A. Hsp90: a new player in DNA repair? Biomolecules 2015;5:2589-618.

13. Beard JA, Tenga A, Chen T. The interplay of NR4A receptors and the oncogene-tumor suppressor networks in cancer. Cell Signal 2015;27:257-66.

14. Wilson AJ, Liu AY, Roland J, et al. TR3 modulates platinum resistance in ovarian cancer. Cancer Res 2013;73:4758-69.

15. Yin P, Wang W, Zhang Z, et al. Wnt signaling in human and mouse breast cancer: focusing on Wnt ligands, receptors and antagonists. Cancer Sci 2018;109:3368-75.

16. Lu H, Huan C. Transcription factor NFAT, its role in cancer development, and as a potential target for chemoprevention. Curr Cancer Drug Targets 2007;7:343-53.

17. Kim JY, Lee JY. Targeting tumor adaption to chronic hypoxia: implications for drug resistance, and how it can be overcome. Int $J$ Mol Sci 2017;18.

18. Dia VP, Krishnan HB. BG-4, a novel anticancer peptide from bitter gourd (Momordica charantia), promotes apoptosis in human colon cancer cells. Sci Rep 2016;6.

19. Fu L-lei, Wen X, Bao J-ku, et al. MicroRNA-modulated autophagic signaling networks in cancer. Int J Biochem Cell Biol 2012;44:733-6.

20. Madden E, Logue SE, Healy SJ, et al. The role of the unfolded protein response in cancer progression: from oncogenesis to chemoresistance. Biol Cell 2019;111:1-17.

21. Xiang J, Zhou L, Zhuang Y, et al. Lactate dehydrogenase is correlated with clinical stage and grade and is downregulated by si-SATB1 in ovarian cancer. Oncol Rep 2018;40:2788-97.

22. Matulonis UA, Shapira-Frommer R, Santin A, et al. Antitumor activity and safety of pembrolizumab in patients with advanced recurrent ovarian cancer: interim results from the phase 2 KEYNOTE-100 study. J Clin Oncol 2018;36(15_suppl).

23. Hamanishi J, Mandai M, Ikeda T, et al. Safety and antitumor activity of anti-PD-1 antibody, nivolumab, in patients with platinum-resistant ovarian cancer. J Clin Oncol 2015;33:4015-22.
24. Disis ML, Patel MR, Pant S, et al. Avelumab (MSB0010718C), an anti-PD-L1 antibody, in patients with previously treated, recurrent or refractory ovarian cancer: a phase $\mathrm{lb}$, open-label expansion trial. $J$ Clin Oncol 2015;33(15_suppl). abstr 5509.

25. Liu YL, Zamarin D. Combination immune checkpoint blockade strategies to maximize immune response in gynecological cancers. Curr Oncol Rep 2018;20. 94.Pmc6244932.

26. Pujade-Lauraine E, Hilpert F, Weber B, et al. Bevacizumab combined with chemotherapy for platinum-resistant recurrent ovarian cancer: the AURELIA open-label randomized phase III trial. J Clin Oncol 2014;32:1302-8.

27. Favaudon V, Caplier L, Monceau V, et al. Ultrahigh dose-rate flash irradiation increases the differential response between normal and tumor tissue in mice. Sci Trans/ Med 2014;6.

28. Atlas TCG. Integrated genomic analyses of ovarian carcinoma. Nature 2011;474:609-15.

29. Mayer MP, Gierasch LM. Recent advances in the structural and mechanistic aspects of hsp70 molecular chaperones. J Biol Chem 2018;294:2085-97.

30. Caumanns JJ, Wisman GBA, Berns K, et al. ARID1A mutant ovarian clear cell carcinoma: a clear target for synthetic lethal strategies. Biochimica et Biophysica Acta (BBA) - Reviews on Cancer 2018;1870:176-84

31. Deng J, Wang L, Chen H, et al. Targeting epithelial-mesenchymal transition and cancer stem cells for chemoresistant ovarian cancer. Oncotarget 2016;7:55771-88.

32. Liu Y, Myrvang HK, Dekker LV. Annexin A2 complexes with $\mathrm{S} 100$ proteins: structure, function and pharmacological manipulation. $\mathrm{Br} J$ Pharmacol 2015;172:1664-76.

33. Cheng C-W, Tse E. Pin1 in cell cycle control and cancer. Front Pharmacol 2018;9:1367.

34. Young AN, Herrera D, Huntsman AC, et al. Phyllanthusmin derivatives induce apoptosis and reduce tumor burden in highgrade serous ovarian cancer by late-stage autophagy inhibition. $\mathrm{Mol}$ Cancer Ther 2018;17:2123-35.

35. Kushnir VA, Seifer DB, Barad DH, et al. Potential therapeutic applications of human anti-müllerian hormone $(\mathrm{AMH})$ analogues in reproductive medicine. J Assist Reprod Genet 2017;34:1105-13.

36. Singh B, Carpenter G, Coffey RJ. EGF receptor ligands: recent advances. F1000Res 2016;5. 